\title{
Chemical Printing of Biological Tissue by Gold Nanoparticle-Assisted Laser Ablation
}

\author{
Siu-Leung Chau, Ho-Wai Tang, Yu-Hong Cheng, Chun-Nam Lok, and Kwan-Ming Ng*(i) \\ Department of Chemistry, The University of Hong Kong, Pokfulam Road, Hong Kong SAR, People's Republic of China
}

Supporting Information

ABSTRACT: A chemical printing method based on gold nanoparticle (AuNP)-assisted laser ablation has been developed. By rastering a thin layer of AuNPs coated on a rat kidney tissue section with a UV laser, biomolecules are extracted and immediately transferred/printed onto a supporting glass substrate. The integrity of the printed sample is preserved, as revealed by imaging mass spectrometric analysis. By studying the mechanism of the extraction/printing process, transiently molten AuNPs were found to be involved in the process, as supported by the color and morphological changes of the AuNP thin film. The success of this molecular printing method was based on the efficient laser-nanomaterial interaction, that is, the strong photoabsorption, laser-induced heating, and phase-transition properties of the AuNPs. It is anticipated that the molecular printing method can be applied to perform site-specific printing, which extracts and transfers biochemicals from different regions of biological tissue sections to different types of supporting materials for subsequent biochemical analysis with the preservation of the original tissue samples.

\section{INTRODUCTION}

Extraction constitutes a critical task for chemical analysis in different aspects, such as food and water analysis, botanical analysis, and pharmaceutical and biomedical analysis. ${ }^{1-3}$ The analytes embedded in samples are required to be isolated and concentrated for chemical analysis. Conventional extraction methods, including solvent extraction, supercritical fluid extraction, and solid-phase extraction, are applied to different analytical purposes. $^{4-6}$ Solvent extraction is the most common approach but possesses several limitations. Specifically, it involves a series of procedures (i.e., sample homogenization, dispersion, and shaking) and also requires proper selection of the solvent that can achieve a balance between extraction efficiency and matrix interference. ${ }^{7}$ More importantly, however, the spatial distribution of the analyte molecules cannot be reconstructed after solvent extraction. The delocalization of biomolecules results in the loss of critical bioinformation, such as the histochemical distribution of biomarkers and localization of drug metabolites in target organs.

Recently, researchers have developed a solid-state extraction/ printing method for biological tissues based on picosecond infrared laser ablation. ${ }^{8-11}$ Biomolecules, such as peptides/ proteins, were extracted upon irradiation by picosecond infrared laser pulse. Water in the biological tissues would absorb the infrared laser energy via the $\mathrm{O}-\mathrm{H}$ bond vibrations. This absorbed energy was then converted to thermal energy, which can heat up the irradiated region instantaneously and facilitate molecular extraction and transfer. ${ }^{12}$ The solid-state extraction/printing process can retain the localization of analyte

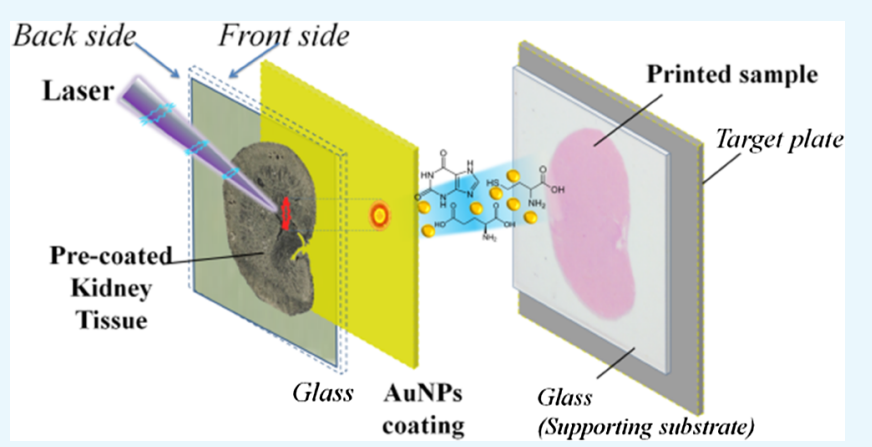

molecules for subsequent analysis, which is particularly useful for the biological tissue analysis and cannot be achieved by conventional solvent extraction. However, the use of an infrared laser would require the presence of water in the sample, which may not be generally practical for dry or preserved samples. Furthermore, infrared lasers are less available, unlike ultraviolet (UV) lasers, which are commonly found in laser desorption/ ionization mass spectrometers. Therefore, it would be advantageous to develop a laser-based solid-state extraction/printing technique using a UV laser.

An in situ solid-state extraction/printing method based on the interaction of a UV laser with AuNPs was developed in the current study. AuNPs with a strong UV absorption property can serve as an efficient media for harvesting the laser energy and extracting the biomolecules from a rat kidney tissue section. Using the solvent-free argon-ion sputtering technique, AuNPs can be homogeneously deposited on the tissue sample surface to form a thin-film layer. ${ }^{13}$ Strong photoabsorption of AuNPs arose from the resonant oscillation of the surface electrons of the AuNPs, which is known as surface plasmon resonance (SPR). ${ }^{14-19}$ After the absorption of laser photons via electronic interband and/or intraband transitions, ${ }^{20-24}$ AuNPs can convert the photon energy to thermal energy. Laserinduced heating, phase transition, or even explosion of the AuNPs would occur if a significant amount of laser energy was

Received: June 26, 2017

Accepted: September 8, 2017

Published: September 21, 2017 
deposited, which has been suggested to promote the desorption process of the analyte molecules. ${ }^{22-24}$ The nanosecond time frame superheating and the transient molten state of the noble metal nanoparticles upon laser irradiation could possibly drive out the molecules from the interior of the sample and promote an in situ solid-state extraction/printing process. Abundant analyte molecules could then be extracted and transferred from the tissue section to a smooth supporting substrate. The integrity of the printed sample can be revealed by performing an imaging mass spectrometric analysis. Here, various kidney metabolites have been detected in the printed samples, with spatial distributions matched with those in the original kidney tissue section. The results of this proof-of-principle study demonstrated the feasibility of the solid-state sample extraction method using the interaction of a UV laser with AuNPs for performing the chemical printing of biological tissue sections.

\section{RESULTS AND DISCUSSION}

Chemical Printing by AuNP-Assisted Laser Ablation. A AuNP-precoated kidney tissue section and two clean glass slides were arranged in a sandwich-like setup (Figure 1). Upon

(a) Schematic diagram of the setup for molecular extraction/printing

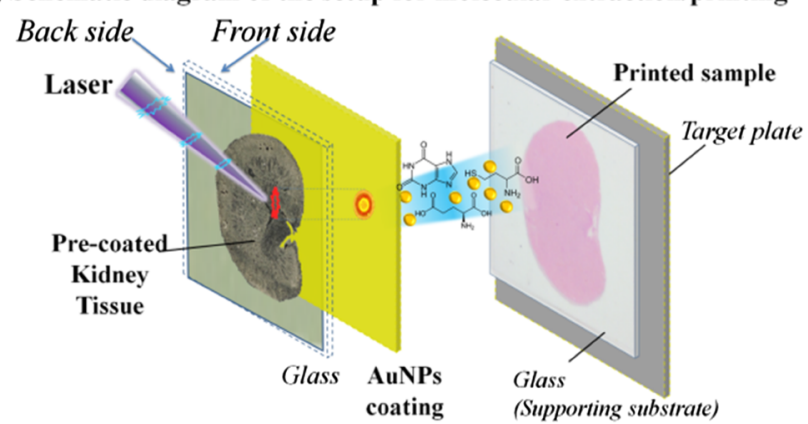

(b) Cross section of the setup

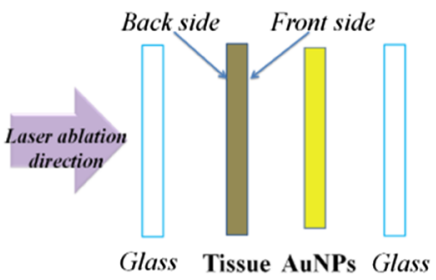

Figure 1. (a) Schematic diagram of the experimental setup for molecular printing by gold nanoparticle (AuNP)-assisted laser ablation. (b) Cross section of the setup showing a detailed arrangement of the laser pulse, a rat kidney section, a thin layer of AuNP coating, and the supporting substrate.

laser irradiation from the back side, the analytes were ejected from the front side of the tissue section and transferred to the clean glass slide (i.e., supporting substrate) to form the molecular transferred sample (i.e., printed sample). In addition, the printed sample exhibited a pale pink color. To identify the analyte molecules transferred in the printed sample, AuNPassisted laser desorption/ionization mass spectrometry was performed. The abundant analyte molecules were detected from the printed sample, although they were not all identified in this proof-of-principle experiment. The mass spectrum with an $m / z$ range of $20-500$ is shown in Figure 2. An accurate mass analysis was performed to identify the compounds detected from the printed sample. Four metabolite ions were identified, and the results are summarized in Table 1 . The metabolite ions at $\mathrm{m} / z 132.0335$ and 146.0484 were identified as [aspartic acid $-\mathrm{H}]^{-}(28.8 \mathrm{ppm})$ and [glutamic acid $\left.-\mathrm{H}\right]^{-}(21.2 \mathrm{ppm})$, respectively. Moreover, deprotonated [hypoxanthine $-\mathrm{H}]^{-}$ and $[\text { xanthine }-\mathrm{H}]^{-}$were the metabolite ions detected at $\mathrm{m} / \mathrm{z}$ $135.0305(1.5 \mathrm{ppm})$ and $151.0261(3.3 \mathrm{ppm})$, respectively. The identification of the detected biochemical as compared with reference standards was further confirmed by tandem mass spectrometric analysis as shown in Table S1.

To examine the integrity of the printed sample, imaging mass spectrometric analysis was conducted. Molecular ion images of the four identified metabolite ions are shown in Figure 3. The metabolite ions of [aspartic acid $-\mathrm{H}]^{-}(m / z$ 132.0) and [glutamic acid $-\mathrm{H}]^{-}(\mathrm{m} / z$ 146.0 $)$ were evenly distributed on the entire kidney tissue section. Deprotonated hypoxanthine $(\mathrm{m} / z$ 135.0) was mainly located at the medulla and pelvis regions of the kidney section, whereas the metabolite ion of $[\text { xanthine }-\mathrm{H}]^{-}(\mathrm{m} / z$ 151.0) was mainly located on the cortex region. Some other metabolites with clear histological distributions, including those at $m / z 92, m / z 134, m / z 258$, $m / z 457$, and $m / z 473$, could also be detected, as shown in Figure S1, although they were not identified in this proof-ofprinciple experiment. In general, histological distributions of the metabolite ions in the printed sample resembled those of the tissue (Figure 4), which revealed that the delocalization of biomolecules during the printing process was insignificant. It is believed that a small laser ablation area and a close distance between the tissue and the supporting substrate would prevent the delocalization of biomolecules during the printing process, and thus the integrity of the printed sample was well-preserved.

In addition, it was noted that the molecular printing could not be achieved without applying the AuNPs, as shown in Figure 5. In a control experiment, only half of a kidney tissue section was coated with a thin layer of AuNPs. After laser irradiation, a pale pink color was only observed in the sample region coated with AuNPs, whereas the control region without the AuNP coating remained colorless (Figure 5a). Furthermore, the printed sample was only observed on the supporting substrate corresponding to the AuNP-coated tissue section region (Figure $5 \mathrm{~b}$ ). In the subsequent imaging mass spectrometry (IMS) analysis of the printed sample, selected molecular ions (including those at $m / z 132,135,146$, and 151) could only be detected from the pink region of the printed sample, as shown in Figure 5c. The results revealed that a thin layer of AuNPs were essential for the printing process.

This chemical printing method could also be applied to transfer the biomolecules from biological tissues to other types of supporting materials, such as nitrocellulose membrane and paper, as shown in Figure $6 a-c$, with the preservation of the original tissue sample. The printed sample on the nitrocellulose membrane and paper substrates could then be analyzed using other biochemical approaches, though it remains to be explored. Moreover, another distinctive advantage of the printing method is its capability of site-specific printing for the selected regions of the tissue section, as shown in Figure 6d, in which the cortex, medulla, and pelvis regions of the kidney section could be separately printed on the different supporting substrate. The printing method is similar to the technique of laser capture microdissection ${ }^{25}$ for the isolation of the targeted site from tissue samples.

Proposed Mechanism of AuNP-Assisted Chemical Printing. We anticipated that, upon laser irradiation, AuNPs would undergo laser-induced heating, followed by phase transition, such as melting, as reported in our previous 
(a) Optical image of printed sample

(b) Mass spectrum in negative mode
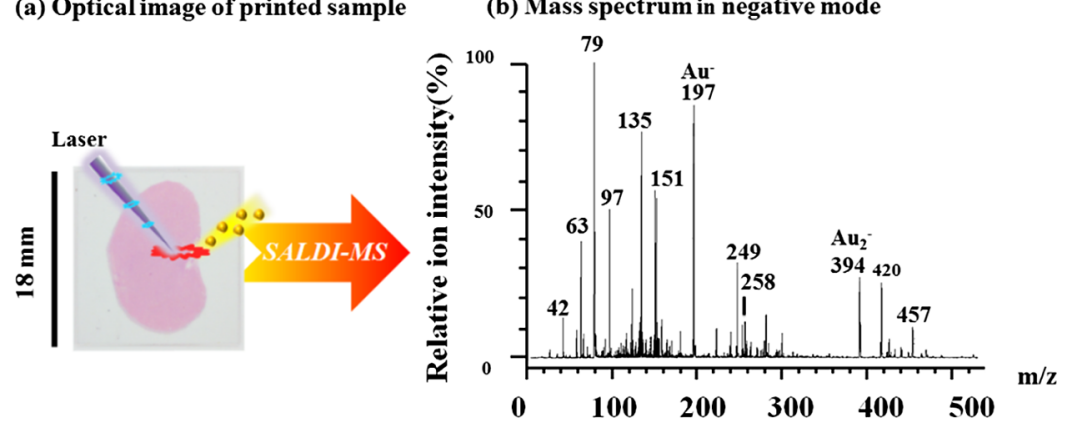

Figure 2. (a) Optical image and (b) surface-assisted laser desorption/ionization mass spectrometry (SALDI-MS) spectrum of the printed sample extracted from the rat kidney tissue section. The kidney tissue was mounted on a glass slide (thickness: $0.13-0.16 \mathrm{~mm}, 18 \times 18 \mathrm{~mm}$ ). The scale bar of the optical image was shown, and it can be applied to all optical images of kidney tissue sections shown in the article.

Table 1. Metabolite Ions Identified in the Printed Sample ${ }^{a, b}$

$\begin{array}{cccc}\begin{array}{c}m / z_{\text {theoretical }} \\ {[\mathrm{M}-\mathrm{H}]^{-}}\end{array} & \begin{array}{c}m / z_{\text {measured }} \\ {[\mathrm{M}-\mathrm{H}]^{-}}\end{array} & \begin{array}{c}\text { mass difference } \\ (\mathrm{ppm})\end{array} \\ \begin{array}{c}\text { aspartic acid } \\ \left(\mathrm{C}_{4} \mathrm{H}_{7} \mathrm{NO}_{4}\right)\end{array} & 132.0296 & 132.0335 & 28.8 \\ \begin{array}{c}\text { hypoxanthine } \\ \left(\mathrm{C}_{5} \mathrm{H}_{4} \mathrm{~N}_{4} \mathrm{O}\right)\end{array} & 135.0307 & 135.0305 & 1.5 \\ \begin{array}{c}\text { glutamic acid } \\ \left(\mathrm{C}_{5} \mathrm{H}_{9} \mathrm{NO}_{4}\right)\end{array} & 146.0453 & 146.0484 & 21.2 \\ \begin{array}{c}\text { xanthine } \\ \left(\mathrm{C}_{5} \mathrm{H}_{4} \mathrm{~N}_{4}\right)\end{array} & 151.0256 & 151.0261 & 3.3\end{array}$

${ }^{a}$ Accurate mass measurements were performed for the ions detected from the printed sample. The mass scale of the instrument was calibrated using gold cluster ion peaks $[\mathrm{Au}]^{-},\left[\mathrm{Au}_{2}\right]^{-}$, and $\left[\mathrm{Au}_{3}\right]^{-}$at $\mathrm{m} / z$ 196.9671, 393.9337, and 590.9002, respectively. ${ }^{{ }}$Tandem MS data are shown in Table S1 of the Supporting Information.

study $^{22-24}$ and shown in a schematic diagram (Figure S2), illustrating its effect on the molecular extraction/printing process. Cell membranes would be destroyed by the heat generated from AuNPs, and biomolecules embedded in the tissue would be extracted and trapped by the transiently molten AuNPs. In fact, the melting of AuNP thin films after laser irradiation was evidenced from the scanning electron microscopic (SEM) examination result, as shown in Figure 7. Prior to laser irradiation, a thin film of AuNPs was uniformly coated on the tissue surface (Figure 7d). However, after laser irradiation, spherical nanoparticles were generated (Figure 7e). The formation of spherical nanoparticles after laser irradiation was also observed for the AuNP thin-film coating with different thicknesses (Figure S3). The morphological change of AuNP coating indicated that the phase transition occurred during the molecular extraction/printing process upon laser irradiation. In addition, spherical nanoparticles could also be observed on the surface of the printed sample, as shown in Figure $7 f$, which revealed the role of AuNPs for carrying the biomolecules to the supporting substrates during the molecular extraction/printing process. Apart from the SEM result, the significant color change of the AuNP coating on the tissue sample also implicated the morphological change of the AuNP thin film during the extraction/printing process. As shown in Figure 7a,b, the AuNP coating changed from slight blue-green (prior to laser irradiation) to pale pink (after laser irradiation). It is wellreported that the color change of AuNPs is related to the plasmonic resonance property, which is highly dependent on the change of particle morphology and size. ${ }^{1,26}$ As shown in the inset of Figure 7e, some smaller AuNPs were formed on the laser-ablated tissue, and more smaller AuNPs were transferred to the printed sample (inset of Figure $7 \mathrm{f}$ ), which could account for the color change of the tissue (from blue-green to pink) and support the transfer of materials to the printed sample. Because the majority of the laser photoenergy has already been modulated by the AuNPs, it is believed that the laser would have minimal effect(s) to the integrity of the analyte molecules/ biological tissue.

Effect of Laser Fluence on Chemical Printing. Previous studies revealed that high laser fluence could induce high laserinduced heating and subsequently cause a higher extent of phase transition. ${ }^{22-24}$ It is anticipated that the higher extent of laser-induced heating/phase transition of AuNPs could result in higher molecular extraction efficiency, and thus more biomolecules could be transferred to/printed on the supporting substrate. Laser fluence between 67 and $126 \mathrm{~mJ} / \mathrm{cm}^{2}$ (maximum fluence) using a constant thickness of AuNP thinfilm coating $(5.0 \mathrm{~nm})$ was adopted to investigate the effect of

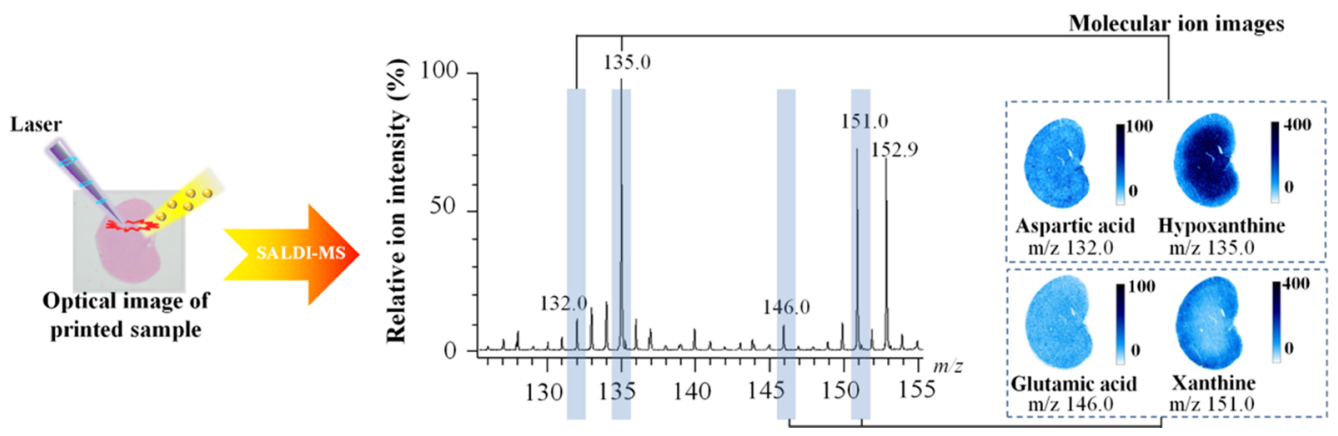

Figure 3. Spatial distributions of four deprotonated metabolite ions in the printed sample revealed by imaging mass spectrometry, including aspartic acid, hypoxanthine, glutamic acid and xanthine. 
Selected molecular ion images
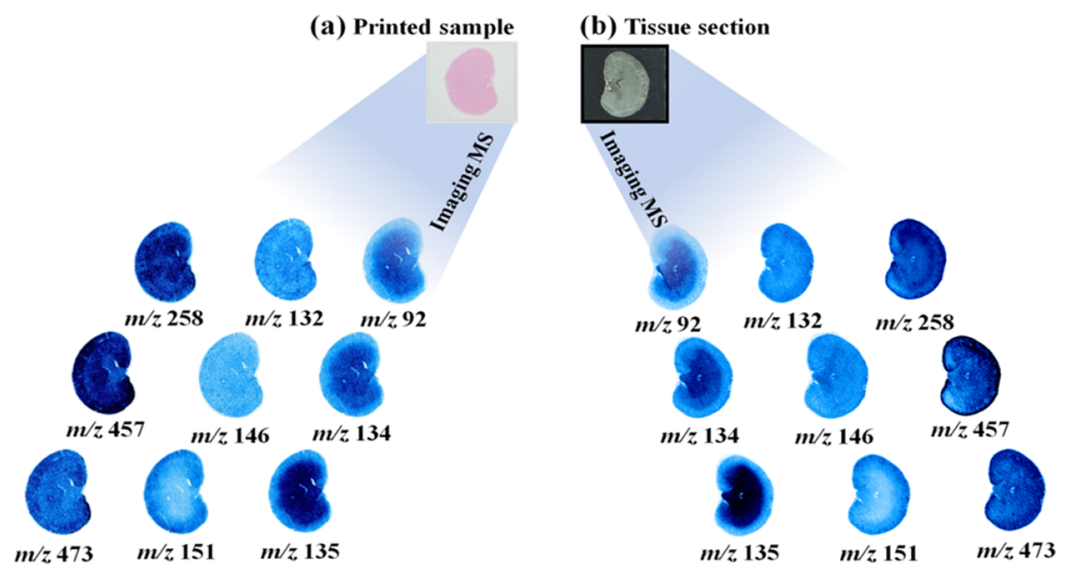

Figure 4. Preservation of the histochemical information in the printed sample. Spatial distributions of the nine selected metabolite ions at $m / z$, $132,134,135,146,151,258,457$, and 473 in the (a) printed sample were similar to those in the (b) original rat kidney tissue section.

\section{Optical image of tissue section and printed sample}

(a) Kidney tissue section after laser ablation

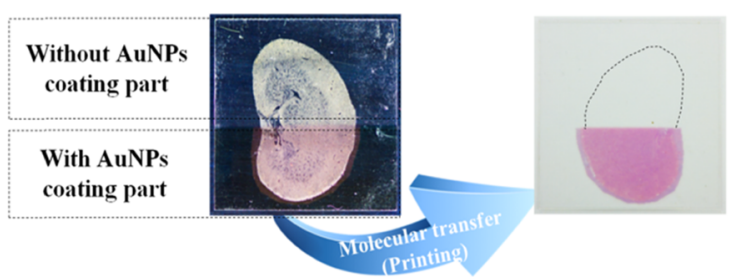

(c) Selected molecular ion images

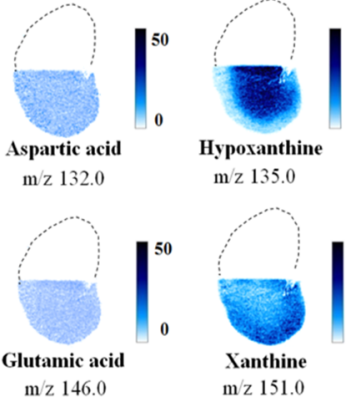

Figure 5. Essential role of AuNP coating in the molecular extraction/printing demonstrated by a control experiment. (a) After laser irradiation, only the lower half of the kidney tissue section, which was coated with AuNPs, appeared pink, whereas no observable change was noted for the upper half without the AuNP coating. Furthermore, printed sample was only observed for the lower half of the tissue section, as shown in (b). In addition, (c) metabolite ions were only detected in the lower half (i.e., pink region) of the printed sample by using imaging mass spectrometry.
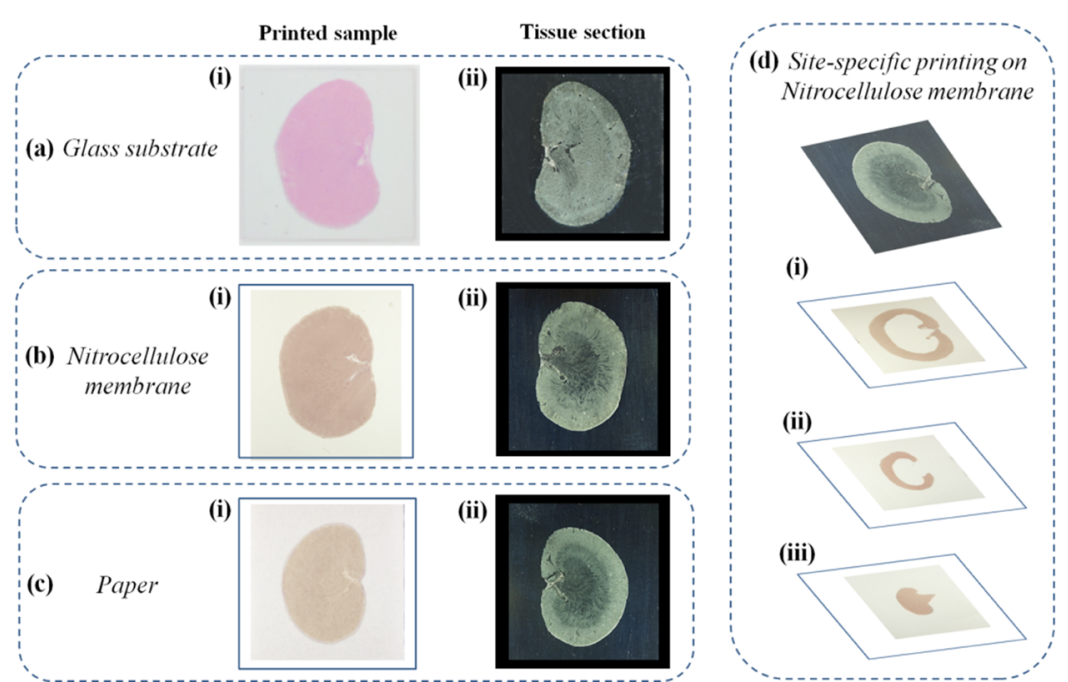

Figure 6. Chemical printing of rat kidney tissue sections on different types of supporting materials: (a) glass, (b) nitrocellulose membrane, and (c) paper, with the preservation of the original tissue sections and (d) site-specific printing of the tissue section: (d-i) cortex region, (d-ii) medulla region, and (d-iii) pelvis region on the nitrocellulose membrane.

laser fluence on molecular extraction/printing efficiency. Ion intensities of five selected characteristic metabolite ions with small-to-medium mass range, including the ions at $m / z 92,135$, 258,457 , and 473 with clear histological distributions, detected from the printed sample were plotted against the laser fluence, as shown in Figure 8a. The results revealed that the ion intensities of selected ions increased gradually with laser fluence from 67 to $111 \mathrm{~mJ} / \mathrm{cm}^{2}$ and became steady when the laser 


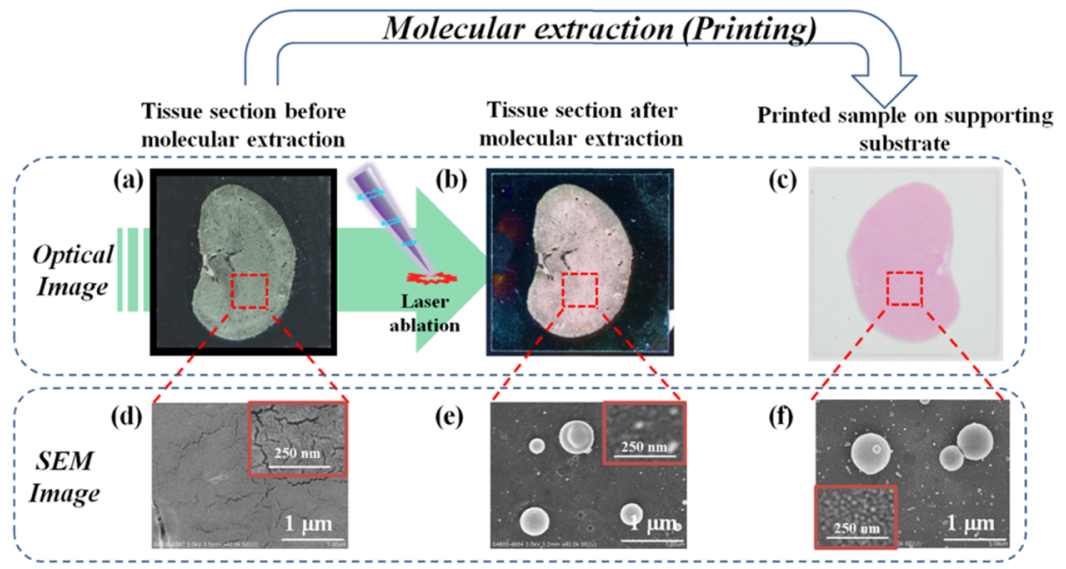

Figure 7. Phase change (i.e., melting) of the AuNP thin film during the molecular extraction/printing process, as supported by the optical color and morphological changes before and after laser irradiation. The color of the AuNP coating on the kidney tissue section changed from (a) bluish green to (b) pink after molecular extraction. From the scanning electron micrograph, (d) the AuNP coating was even and homogenous before laser irradiation. However, after laser irradiation, spherical AuNPs were (e) generated on the tissue section and (f) transferred to the printed sample, which revealed a phase change of the AuNP thin film and accounted for (c) the pink color of the printed sample. The smaller AuNPs responsible for the pink color are shown in the insets (red box) of $(e, f)$.

(a) Effect of laser fluence on molecular extraction (Thickness: $5.0 \mathrm{~nm}$ )

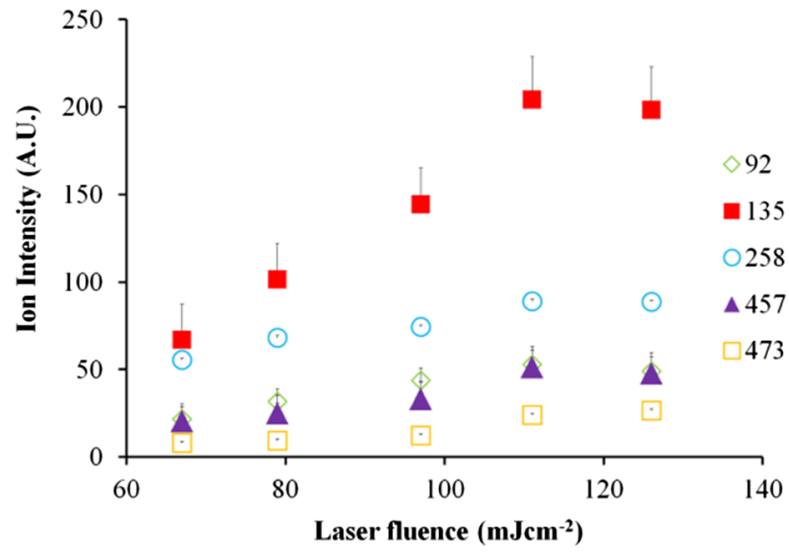

(b) Effect of thickness of AuNPs coating on molecular extraction (Laser fluence: $111 \mathrm{~mJ} / \mathrm{cm}^{2}$ )

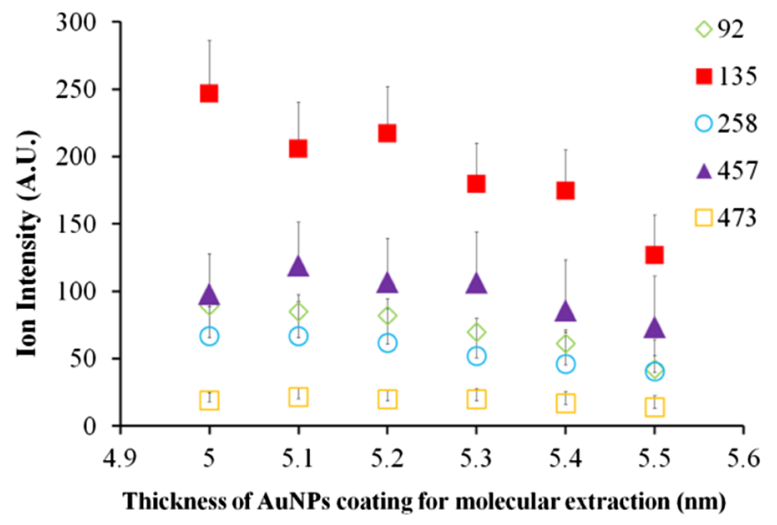

Figure 8. Effects of laser fluence and thickness of AuNP coating on the molecular extraction/printing efficiency. In general, the efficiency was found to (a) increase with laser fluence and (b) decrease with AuNP coating thickness, based on the measured intensities of five selected metabolite ions.

fluence was beyond $111 \mathrm{~mJ} / \mathrm{cm}^{2}$. The increasing trend of ion intensities might be because of higher molecular extraction efficiency, as a consequence of higher laser-induced heating and extent of phase transition. In addition, different extraction efficiencies were observed among the five selected metabolite ions, which could be because of their different binding interaction with the transiently molten AuNPs during the extraction process. A SEM examination was carried out, and the result was shown in Figure S4. It was observed that the amount of spherical nanoparticles formed on the printed sample was found to increase with the applied laser fluence. The average number of spherical nanoparticles increased from $42 \pm 13$ to $108 \pm 17$ when the laser fluence applied to the molecular extraction/printing increased from 67 to $126 \mathrm{~mJ} / \mathrm{cm}^{2}$ (Figure S4). The increase was statistically significant at the confidence level of $90 \%$ (based on the Student's $t$-test). The increasing amount of spherical nanoparticles generated could be attributed to the higher degree of superheating and the extent of melting of AuNPs. Hence, more biomolecules could be extracted and transferred to/printed on the supporting substrate with the assistance of more newly formed spherical nanoparticles. Thus, an increasing trend of molecular extraction/printing efficiency could be observed with the increasing laser fluence (Figure 8a).

Effect of Thickness of AuNP Coating on Chemical Printing. As shown in Figure $8 \mathrm{~b}$, the ion intensities of the five selected characteristic metabolite ions (including $m / z 92,135$, 258,457 , and 473) detected from the printed sample were plotted against the thickness of AuNP thin-film coating (from 5.0 to $5.5 \mathrm{~nm}$ ) using a constant laser fluence of $111 \mathrm{~mJ} / \mathrm{cm}^{2}$. An opposite trend between the molecular extraction/printing efficiency and the thickness of the thin film was observed, that is, the thinnest coating, $5.0 \mathrm{~nm}$, resulted in the highest extraction efficiency and vice versa. From the SEM examination results, the sizes of those spherical nanoparticles formed after laser irradiation were found to increase with the thickness of AuNP coating (Figure S5). As shown in Figure S5b,c, for the smallest film thickness (i.e., $5.0 \mathrm{~nm}$ ), the majority of AuNPs generated on the tissues after laser irradiation was $<200 \mathrm{~nm}$ in diameter and contributed to $70 \%$ of the total particle coverage area. When the film thickness increased to $5.3 \mathrm{~nm}$, the size of the AuNPs increased in general and some of them were 300$400 \mathrm{~nm}$ in diameter. When the film thickness increased to 5.5 $\mathrm{nm}$, some much larger AuNPs (>400 nm in diameter) appeared and dominated the total particle coverage area $(>70 \%)$. The 
trend of the particle sizes was inversely correlated with molecular extraction efficiencies, that is, the larger the laserinduced spherical nanoparticles that were formed, the lower the ion intensities that were detected. A possible reason for this could be the relatively larger proportion of the extracted biomolecules that was embedded in the internal volume of the newly generated spherical AuNPs, especially for larger spherical AuNPs which have a smaller surface-to-volume ratio. Hence, in the subsequent SALDI-MS analysis of the printed sample, larger spherical AuNPs could not desorb most of the biomolecules embedded in the internal volume, whereas smaller spherical AuNPs having a higher surface-to-volume ratio could desorb the extracted biomolecules more readily. ${ }^{27}$ Overall, the results demonstrated that higher laser fluence and thinner AuNP coating were more favorable for the efficient extraction/printing process.

\section{CONCLUSIONS}

A novel chemical printing method for a biological tissue based on AuNP-assisted laser ablation was developed. Biomolecules from a rat kidney tissue section have been successfully extracted and printed onto a supporting substrate. Imaging mass spectrometric analysis showed that the distribution of biomolecules in the printed sample was similar to the histochemical distribution of the metabolites in the original kidney tissue sections, showing that the integrity of the printed sample was well-preserved in the printing process. The printing process utilized efficient photoabsorption, laser-induced heating, and phase-transition properties of AuNPs. The effects of laser fluence and thickness of the AuNP thin film on the extraction/printing efficiency were systematically investigated. In the mechanistic study, upon laser irradiation, a thin layer of AuNPs on the tissue section would undergo rapid heating and become transiently molten, which could facilitate the extraction of biomolecules embedded in the tissue. The extracted biomolecules would then be engulfed into the resolidified AuNPs, and subsequently transferred to/printed on the opposite supporting substrate because of the thermal and/or pressure gradient generated by laser irradiation. The findings of this proof-of-principle study demonstrated that the molecular printing of biological tissues can be achieved by employing AuNPs and a UV laser. This printing technique would be suitable for handling tiny samples (e.g., tissue sections and biopsies), which might be challenging for conventional solvent extraction. Moreover, the developed chemical printing technique will be able to transfer the biomolecules from sitespecific regions of a biological tissue section to different types of supporting materials, such as nitrocellulose membrane or paper, for other subsequent biochemical analysis with the preservation of the original tissue samples.

\section{EXPERIMENTAL SECTION}

Chemicals. Aspartic acid, hypoxanthine, glutamic acid, and xanthine were purchased from Sigma-Aldrich (St. Louis, MO, USA) with a purity of $95 \%$ or above.

Animal Tissue and Sample Preparation. Animal tissues were collected in compliance with animal use and care regulation of The University of Hong Kong. Frozen kidney tissues of a male Sprague Dawley rat were cryosectioned (12 $\mu \mathrm{m}$ thickness) at $-20{ }^{\circ} \mathrm{C}$ chamber temperature using a cryostat (FE/FSE, Thermo Fisher Scientific, Inc. Waltham, MA, USA) and thaw-mounted on clean TED glass slides (thickness: 0.13-
$0.16 \mathrm{~mm}, 18 \times 18 \mathrm{~mm}$; Ted Pella, Inc., Redding, CA, USA). The UV-visible absorption spectrum of the TED glass slides in the range of $250-600 \mathrm{~nm}$ is shown in Figure S6. Optimal cutting temperature (OCT) solution was used to fix one side of the sample to the cryostat support. To avoid contamination, particular care was taken to avoid any contact of the OCT solution with the exposed side of the tissue.

Chemical Printing by AuNP-Assisted Laser Ablation. The tissue sections mounted on clean glass slides were then coated with a thin layer of AuNPs using argon-ion sputtering. The sputter coater (SCD 005; Bal-Tec AG, Liechtenstein) was operated using an ultrahigh-purity argon gas (99.999\%) and a high-purity gold target (99.99\%; Ted Pella, Inc., Redding, CA, USA). For the molecular printing, the following sputtering conditions were applied: sputtering current: $30 \mathrm{~mA}$; sputtering time: $30 \mathrm{~s}$; distance between the gold target and the sample: $50-75 \mathrm{~mm}$; and chamber pressure during sputtering: 0.040.06 mbar.

To perform the printing by the AuNP-assisted laser ablation, a clean TED glass slide, which acted as a supporting substrate, was placed on top of the AuNP-coated rat kidney tissue section to form a sandwich-like setup (Figure 1). This sandwich-like setup was then mounted tightly on a modified stainless steel MALDI sample plate using an electrically conductive tape (9713 XYZ-Axis; 3M, St. Paul, MN, USA), with the supporting substrate touching the MALDI sample plate. The indentation $\left(117 \times 71 \mathrm{~mm}^{2}\right.$ in area and $0.2 \mathrm{~mm}$ in depth $)$ of the modified MALDI plate was used to partly compensate for the thickness $(0.26-0.32 \mathrm{~mm})$ of the double-glass-slide setup. The tissue section with a thin layer of AuNP coating was ablated by a pulsed laser (Nd:YAG solid-state smartbeam laser with a wavelength of $355 \mathrm{~nm}$ and pulsed duration of $6 \mathrm{~ns}$, Azura Laser AG, Berlin, Germany) with a firing frequency of $20 \mathrm{~Hz}$ to generate the printed sample on the supporting substrate. The laser shot number for each raster position was set at 30, and the raster step size was set at $70 \mu \mathrm{m}$. After laser ablation, the optical images of the tissue sections and the printed samples were recorded using a digital camera equipped with a macrolens (EOS 550D with EF $100 \mathrm{~mm} \mathrm{f/2.8} \mathrm{Marco} \mathrm{USM,} \mathrm{Canon,}$ Japan).

To elucidate the effects of laser fluence and thickness of AuNP thin-film coating on the molecular-printing process, different laser fluence range and different thickness of AuNP coating were adopted. Laser fluence between 76 and 100\% $\left(67.2-126.4 \mathrm{~mJ} / \mathrm{cm}^{2}\right)$ was used to examine the effect of laser fluence on molecular printing. Each tissue section for the study was coated with the same thickness $(5 \mathrm{~nm})$ of AuNP coating. For studying the effect of thickness of AuNP coating, different thicknesses of AuNP coating were generated by tuning the distances between the gold target and tissue samples (including $75,70,65,60,55$, and $50 \mathrm{~mm}$ ). The thicknesses of the AuNP thin films were determined to be 5.0, 5.1, 5.2, 5.3, 5.4, and 5.5 $\mathrm{nm}$ using a transmission electronic microscope (Figure S7). In addition, UV-visible absorption spectroscopic measurement using a UV-visible spectrophotometer (Cary 60 UV-vis, Agilent Technologies, Santa Clara, CA) was also performed to monitor the increase in the AuNP-coating thickness, as the increment of AuNP-coating thickness would increase the UVvisible absorption path length and thus increase the absorbance generally (Figure S8). Each sample was ablated with a laser at the same laser fluence $\left(111 \mathrm{~mJ} / \mathrm{cm}^{2}\right)$. For both studies, the laser shot number, firing frequency, and raster step size remained the same. 
SALDI-MS was adopted to evaluate the extraction efficiency (i.e., the amount of biomolecules being transferred to the supporting substrate); all of the printed samples were subsequently coated with AuNPs using the same sputtering conditions (sputtering current: $30 \mathrm{~mA}$; sputtering duration: 20 s; sputtering distance: $53 \mathrm{~mm}$; and chamber pressure during sputtering: $0.04-0.06 \mathrm{mbar}$ ). The same conditions of AuNP coating would ensure a fair comparison of the extraction efficiency.

SALDI-TOF Mass Spectrometric Measurement. Ultraflex II MALDI-TOF/TOF MS with a $355 \mathrm{~nm}$ solid-state laser (Bruker Daltonics, Bremen, Germany) was employed for the molecular printing and SALDI-based imaging MS analysis. The mass spectrometer was operated in a negative reflectron mode. The ion source 1 , ion source 2 , lens, reflector 1 , and reflector 2 voltages were set at $20.00,18.42,8.18,21.00$, and $9.62 \mathrm{kV}$, respectively. The detector voltage was set at $1611 \mathrm{~V}$. The ionextraction delay time was set to $850 \mathrm{~ns}$ for SALDI-based imaging MS analysis. The laser fluence was set at a range between 80 and $86 \%\left(89-104 \mathrm{~mJ} / \mathrm{cm}^{2}\right)$, with a firing frequency of $15 \mathrm{~Hz}$. The laser shot number for each raster position was set at 5 , and the raster step size was set at $70 \mu \mathrm{m}$. The effective dimension of the laser spots in a circular shape was $\sim 135 \times 135$ $\mu \mathrm{m}$. The mass spectra were acquired in the $m / z$ range of $20-$ 800. The gold cluster ions $\left(\mathrm{Au}^{-}, \mathrm{Au}_{2}{ }^{-}\right.$, and $\left.\mathrm{Au}_{3}{ }^{-}\right)$generated from the AuNP-coated molecular-transferred sample were employed for internal mass calibration. Instrument control and MS data acquisition were performed using FlexControl software (version 2.4, Bruker Daltonics, Bremen, Germany), and data processing was conducted using FlexAnalysis software (version 1.2, Bruker Daltonics, Bremen, Germany). The IMS data and molecular images were processed and exported using BioMap software (version 3.8.0.3; Novartis, Basel, Switzerland).

Tandem mass spectrometric experiments were performed on a UltrafleXtreme MALDI-TOF/TOF MS (Bruker Daltonics, Bremen, Germany) instrument with a $355 \mathrm{~nm}$ solid-state laser (Nd:YAG solid state smartbeam II laser with pulsed duration of $3 \mathrm{~ns}$ ). LIFT mode was employed for recording the dissociation of metastable ions of selected precursor ions by laser-induced excitation. Precursor-ion-selection window range is $0.45 \%$ of the parent mass. The ion source 1 , ion source 2 , lens, reflector 1 , and reflector 2 voltages were set at 7.49, 6.79, 3.50, 29.52, and $14.00 \mathrm{kV}$, respectively. The LIFT 1 and LIFT 2 voltages were set at 19.00 and $3.95 \mathrm{kV}$, respectively. The laser power was set to $50-80 \%$ with a firing frequency of $100 \mathrm{~Hz}$. The ionextraction delay time was set to $90 \mathrm{~ns}$. The detector voltage was set at $2391 \mathrm{~V}$.

Electron Microscopic Examination. A scanning electron microscope (S-4800 field-emission scanning electron microscope, Hitachi High-Technologies, Japan) was employed for the examination of the morphology and the size of AuNP coating on the rat kidney tissue surface (before and after molecular printing) and on the supporting substrates after molecular printing. A transmission electron microscope (Tecnai G2 20 S-TWIN; FEI, Hillsboro, OR, USA) was employed for the examination of the thickness of AuNP thinfilm coating.

\section{ASSOCIATED CONTENT}

\section{S Supporting Information}

The Supporting Information is available free of charge on the ACS Publications website at DOI: 10.1021/acsomega.7b00385.
Results of tandem mass spectrometric analysis, additional molecular-ion images, schematic diagram of the proposed mechanism, additional SEM and TEM images, and UVvisible absorbance of AuNP coating with different thicknesses (PDF)

\section{AUTHOR INFORMATION}

\section{Corresponding Author}

*E-mail: kwanmng@hku.hk. Phone: (852)-22194696 (K.M.N.).

\section{ORCID}

Kwan-Ming Ng: 0000-0003-4353-9658

\section{Author Contributions}

The manuscript was written through contributions of all authors. All authors have given approval to the final version of the manuscript.

\section{Notes}

The authors declare no competing financial interest.

\section{ACKNOWLEDGMENTS}

We thank Frankie Y. F. Chan of the Electron Microscope Unit of The University of Hong Kong for assisting in the SEM measurements. K.-M.N. acknowledges the funding support of the General Research Fund (grant no.: HKU_17304014) of the Hong Kong Research Grants Council. K.-M.N. also acknowledges the UGC funding administered by The University of Hong Kong for supporting the Electrospray Ionization Quadrupole Time-of-Flight Mass Spectrometry Facilities under the Support of Interdisciplinary Research in Chemical Science. Moreover, the funding support of The University Research Committee of The University of Hong Kong (project nos.: 201511159200 and 201611159062) is gratefully acknowledged.

\section{REFERENCES}

(1) Palma, M.; Taylor, L. T.; Zoecklein, B. W.; Douglas, L. S. Supercritical Fluid Extraction of Grape Glycosides. J. Agric. Food Chem. 2000, 48, 775-779.

(2) Milstein, L. S.; Essader, A.; Murrell, C.; Pellizzari, E. D.; Fernando, R. A.; Raymer, J. H.; Akinbo, O. Sample Preparation, Extraction Efficiency, and Determination of Six Arsenic Species Present in Food Composites. J. Agric. Food Chem. 2003, 51, 41804184.

(3) Clark, K. D.; Zhang, C.; Anderson, J. L. Sample Preparation for Bioanalytical and Pharmaceutical Analysis. Anal. Chem. 2016, 88, 11262-11270.

(4) Pyo, D.; Shin, H. Supercritical Fluid Extraction of Microcystins from Cyanobacteria. Anal. Chem. 1999, 71, 4772-4775.

(5) Ullah, S. M. R.; Murphy, B.; Dorich, B.; Richter, B.; Srinivasan, K. Fat Extraction from Acid- and Base-Hydrolyzed Food Samples Using Accelerated Solvent Extraction. J. Agric. Food Chem. 2011, 59, 21692174.

(6) Saini, G.; Yang, L.; Lee, M. L.; Dadson, A.; Vail, M. A.; Linford, M. R. Amino-Modified Diamond as a Durable Stationary Phase for Solid-Phase Extraction. Anal. Chem. 2008, 80, 6253-6259.

(7) Murphy, P. A.; Barua, K.; Hauck, C. C. Solvent Extraction Selection in the Determination of Isoflavones in Soy Foods. J. Chromatogr. B: Anal. Technol. Biomed. Life Sci. 2002, 777, 129-138.

(8) Kwiatkowski, M.; Wurlitzer, M.; Omidi, M.; Ren, L.; Kruber, S.; Nimer, R.; Robertson, W. D.; Horst, A.; Miller, R. J. D.; Schlüter, H. Ultrafast Extraction of Proteins from Tissues Using Desorption by Impulsive Vibrational Excitation. Angew. Chem., Int. Ed. 2015, 54, 285-288. 
(9) Ren, L.; Robertson, W. D.; Reimer, R.; Heinze, C.; Schneider, C.; Eggert, D.; Truschow, P.; Hansen, N.-O.; Kroetz, P.; Zou, J.; Miller, R. J. D. Towards Instantaneous Cellular Level Bio Diagnosis: Laser Extraction and Imaging of Biological Entities with Conserved Integrity and Activity. Nanotechnology 2015, 26, 284001.

(10) Donnarumma, F.; Murray, K. K. Laser Ablation Sample Transfer for Localized LC-MS/MS Proteomic Analysis of Tissue. J. Mass Spectrom. 2016, 51, 261-268.

(11) Kwiatkowski, M.; Wurlitzer, M.; Krutilin, A.; Kiani, P.; Nimer, R.; Omidi, M.; Mannaa, A.; Bussmann, T.; Bartkowiak, K.; Kruber, S.; Uschold, S.; Steffen, P.; Lübberstedt, J.; Küpker, N.; Petersen, H.; Knecht, R.; Hansen, N. O.; Zarrine-Afsar, A.; Robertson, W. D.; Miller, R. J. D.; Schlüter, H. Homogenization of Tissues via PicosecondInfrared Laser (PIRL) Ablation: Giving a Closer View on the In-vivo Composition of Protein Species as Compared to Mechanical Homogenization. J. Proteomics 2016, 134, 193-202.

(12) Franjic, K.; Miller, R. J. D. Vibrationally Excited Ultrafast Thermodynamic Phase Transitions at the Water/Air Interface. Phys. Chem. Chem. Phys. 2010, 12, 5225-5239.

(13) Tang, H.-W.; Wong, M. Y.-M.; Lam, W.; Cheng, Y.-C.; Che, C.M.; Ng, K.-M. Molecular Histology Analysis by Matrix-Assisted Laser Desorption/Ionization Imaging Mass Spectrometry Using Gold Nanoparticles as Matrix. Rapid Commun. Mass Spectrom. 2011, 25, 3690-3696.

(14) Barnes, W. L.; Dereux, A.; Ebbesen, T. W. Surface Plasmon Subwavelength Optics. Nature 2003, 424, 824-830.

(15) Homola, J.; Yee, S. S.; Gauglitz, G. Surface Plasmon Resonance Sensors: Review. Sens. Actuators, B 1999, 54, 3-15.

(16) Haes, A. J.; Van Duyne, R. P. A Nanoscale Optical Biosensor: Sensitivity and Selectivity of an Approach Based on the Localized Surface Plasmon Resonance Spectroscopy of Triangular Silver Nanoparticles. J. Am. Chem. Soc. 2002, 124, 10596-10604.

(17) Hecht, B.; Bielefeldt, H.; Novotny, L.; Inouye, Y.; Pohl, D. W. Local Excitation, Scattering, and Interference of Surface Plasmons. Phys. Rev. Lett. 1996, 77, 1889.

(18) Atay, T.; Song, J.-H.; Nurmikko, A. V. Strongly Interacting Plasmon Nanoparticle Pairs: From Dipole-Dipole Interaction to Conductively Coupled Regime. Nano Lett. 2004, 4, 1627-1631.

(19) Kelly, K. L.; Coronado, E.; Zhao, L. L.; Schatz, G. C. The Optical Properties of Metal Nanoparticles: The Influence of Size, Shape, and Dielectric Environment. J. Phys. Chem. B 2003, 107, 668677.

(20) Silina, Y. E.; Volmer, D. A. Nanostructured Solid Substrates for Efficient Laser Desorption/Ionization Mass Spectrometry (LDI-MS) of Low Molecular Weight Compounds. Analyst 2013, 138, 70537065.

(21) Stolee, J. A.; Walker, B. N.; Zorba, V.; Russo, R. E.; Vertes, A. Laser-Nanostructure Interactions for Ion Production. Phys. Chem. Chem. Phys. 2012, 14, 8453-8471.

(22) Lai, S. K.-M.; Tang, H.-W.; Lau, K.-C.; Ng, K.-M. Nanosecond UV Laser Ablation of Gold Nanoparticles: Enhancement of Ion Desorption by Thermal-Driven Desorption, Vaporization, or Phase Explosion. J. Phys. Chem. C 2016, 120, 20368-20377.

(23) Ng, K.-M.; Chau, S.-L.; Tang, H.-W.; Wei, X.-G.; Lau, K.-C.; Ye, F.; Ng, A. M.-C. Ion-Desorption Efficiency and Internal-Energy Transfer in Surface-Assisted Laser Desorption/Ionization: More Implication(s) for the Thermal-Driven and Phase-Transition-Driven Desorption Process. J. Phys. Chem. C 2015, 119, 23708-23720.

(24) Lai, S. K.-M.; Cheng, Y.-H.; Tang, H.-W.; Ng, K.-M. SilverGold Alloy Nanoparticles as Tunable Substrates for Systematic Control of Ion-Desorption Efficiency and Heat Transfer in SurfaceAssisted Laser Desorption/Ionization. Phys. Chem. Chem. Phys. 2017, 19, 20795-20807.

(25) Bhattacharya, S. H.; Gal, A. A.; Murray, K. K. Laser Capture Microdissection MALDI for Direct Analysis of Archival Tissue. J. Proteome Res. 2003, 2, 95-98.

(26) Njoki, P. N.; Lim, I.-I. S.; Mott, D.; Park, H.-Y.; Khan, B.; Mishra, S.; Sujakumar, R.; Luo, J.; Zhong, C.-J. Size Correlation of
Optical and Spectroscopic Properties for Gold Nanoparticles. J. Phys. Chem. C 2007, 111, 14664-14669.

(27) Chiang, C.-K.; Chen, W.-T.; Chang, H.-T. Nanoparticle-based Mass Spectrometry for the Analysis of Biomolecules. Chem. Soc. Rev. 2011, 40, 1269-1281. 